\title{
Risk Factors of Unfavorable Outcomes, Major Bleeding, and All-Cause Mortality in Patients with Venous Thromboembolism
}

\author{
Han Young Lee ${ }^{1}$, Tae Hoon Yeo', Tae Kyung Heo', Young Gyu Cho', Dong Hui Cho', \\ and Kyung Bok Lee ${ }^{2}$ \\ ${ }^{1}$ Department of Surgery, Seoul Medical Center, Seoul, ${ }^{2}$ Department of Surgery, Dongguk University llsan Hospital, Goyang, Korea
}

\begin{abstract}
Purpose: This study aimed to analyze the clinical outcomes of venous thromboembolism (VTE) patients and identify the risk factors for VTE-related unfavorable outcomes, major bleeding, and 30-day all-cause mortality.

Materials and Methods: From January 2016 to December 2020, 198 patients with confirmed VTE were enrolled. Potential risk factors for unfavorable outcomes, major bleeding, and all-cause mortality were analyzed.

Results: VTE-related unfavorable outcomes developed in 13.1\%, while 30-day all-cause mortality was $8.6 \%$. In the multivariate analysis, a pulse $\geq 110 / \mathrm{min}$ and respiratory rate $\geq 30 / \mathrm{min}$ were statistically significant predictors for VTE-related unfavorable outcomes. Diabetes was a significant risk factor for major bleeding. In addition, a history of malignancy, no anticoagulation treatment, and need for mechanical ventilation were significant predictors of all-cause mortality.

Conclusion: VTE-related mortality and morbidity rates remained high. In cases of tachycardia and tachypnea, early aggressive treatment is needed to prevent unfavorable outcomes. Patients with risk factors should be closely monitored.
\end{abstract}

Key Words: Venous thromboembolism, Risk factors, Mortality, Anticoagulants
Received June 14, 2021

Revised August 17, 2021

Accepted November 16, 2021

Published on December 31, 2021

Corresponding author: Kyung Bok Lee Department of Surgery, Dongguk University llsan Hospital, 27 Dongguk-ro, llsandong-gu, Goyang 10326, Korea Tel: 82-2-961-7027 E-mail: md.kblee@outlook.com https://orcid.org/0000-0003-1111-118X

Copyright (c) 2021 The Korean Society for Vascular Surgery

This is an Open Access article distributed under the terms of the Creative Commons Attribution Non-Commercial License (http://creativecommons.org/licenses/by-nc/4.0) which permits unrestricted non-commercial use, distribution, and reproduction in any medium, provided the original work is properly cited.

Cite this article; Vasc Specialist Int 2021. https://doi.org/10.5758/vsi.210041

\section{INTRODUCTION}

Venous thromboembolism (VTE), including deep venous thrombosis (DVT) and pulmonary embolism (PE), is generally considered a common and similar disease entity that expresses different clinical features [1,2]. VTE is a major cause of morbidity and mortality in most Western countries [3]. VTE is the leading cause of preventable early death with appropriate treatment. Hip fracture, major general surgery, major trauma, malignancy/chemotherapy, bed rest $>3$ days, and recent pregnancy (within 3 months of delivery) are well-known risk factors for the occurrence of VTE [4]. The incidence of VTE is lower in Asian countries than in West- ern countries. Several population-based studies have shown that, although the overall incidence of PE is reduced, the average mortality rate remains high at $14 \%$ to $30 \%$ [5].

VTE can be confirmed using computed tomography (CT) for PE and a combination of compression ultrasound (CUS) and CT for DVT. CUS is the most common imaging modality for DVT. For the diagnosis of proximal DVT, CUS shows a sensitivity of $90.1 \%$ and specificity of $97.3 \%$ [6]; however, recent advances in imaging technologies have replaced CUS with CT for diagnosing DVT.

The introduction of anticoagulant therapy reduces VTErelated mortality and morbidity [7]. The recent American College of Chest Physician guidelines recommend at least 
three months of a new oral anticoagulant (NOAC; such as dabigatran, rivaroxaban, apixaban, and edoxaban) alone over warfarin for acute VTE [8]. The advent of acute-phase anticoagulant treatment strategies might improve the clinical outcomes of patients with VTE. The absence of anticoagulation therapy is associated with a 3.2-fold increase in mortality [9].

This study aimed to analyze the clinical outcomes of VTE patients and identify the predictors of VTE-related unfavorable outcomes, such as major bleeding and 30-day allcause mortality.

\section{MATERIALS AND METHODS}

From January 2016 to December 2020, 198 patients with confirmed VTE were enrolled. DVT was diagnosed using CUS or CT venography (CTV). PE was confirmed using CT pulmonary angiography (CTPA). All CTV and CTPA results were elucidated by two board-certified radiologists specializing in vascular imaging.
DVT was classified into proximal or distal. Proximal DVT was defined as a thrombus affecting the popliteal or proximal vein (Fig. 1). Each PE was diagnosed using CTPA (Fig. 2). Additionally, the simplified pulmonary embolism severity index (sPESI) was calculated. A high sPESI was defined as age $>80$ years; systolic blood pressure $<100 \mathrm{mmHg}$; heart rate $>110 \mathrm{bpm} ; \mathrm{O}_{2}$ saturation $<90 \%$; or current diagnosis of cancer, heart failure, or chronic obstructive pulmonary disease (COPD) [10]. Anticoagulation regimens included unfractionatedor low molecular weight heparin followed by oral vitamin $\mathrm{K}$ antagonist or NOACs for at least three months. An international normalized ratio of 1.5 to 2.5 was considered an appropriate therapeutic range.

In this study, patients with confirmed VTE were classified into isolated DVT or PE (PE with or without DVT) groups. Their clinical characteristics and risk factors (age $>70$ years; previous VTE; immobilization $\geq 3$ days; history of trauma or surgery $\leq 4$ weeks prior; history of malignancy and/or chemotherapy, hypertension, diabetes, coronary artery disease, heart failure, chronic kidney disease, cere-
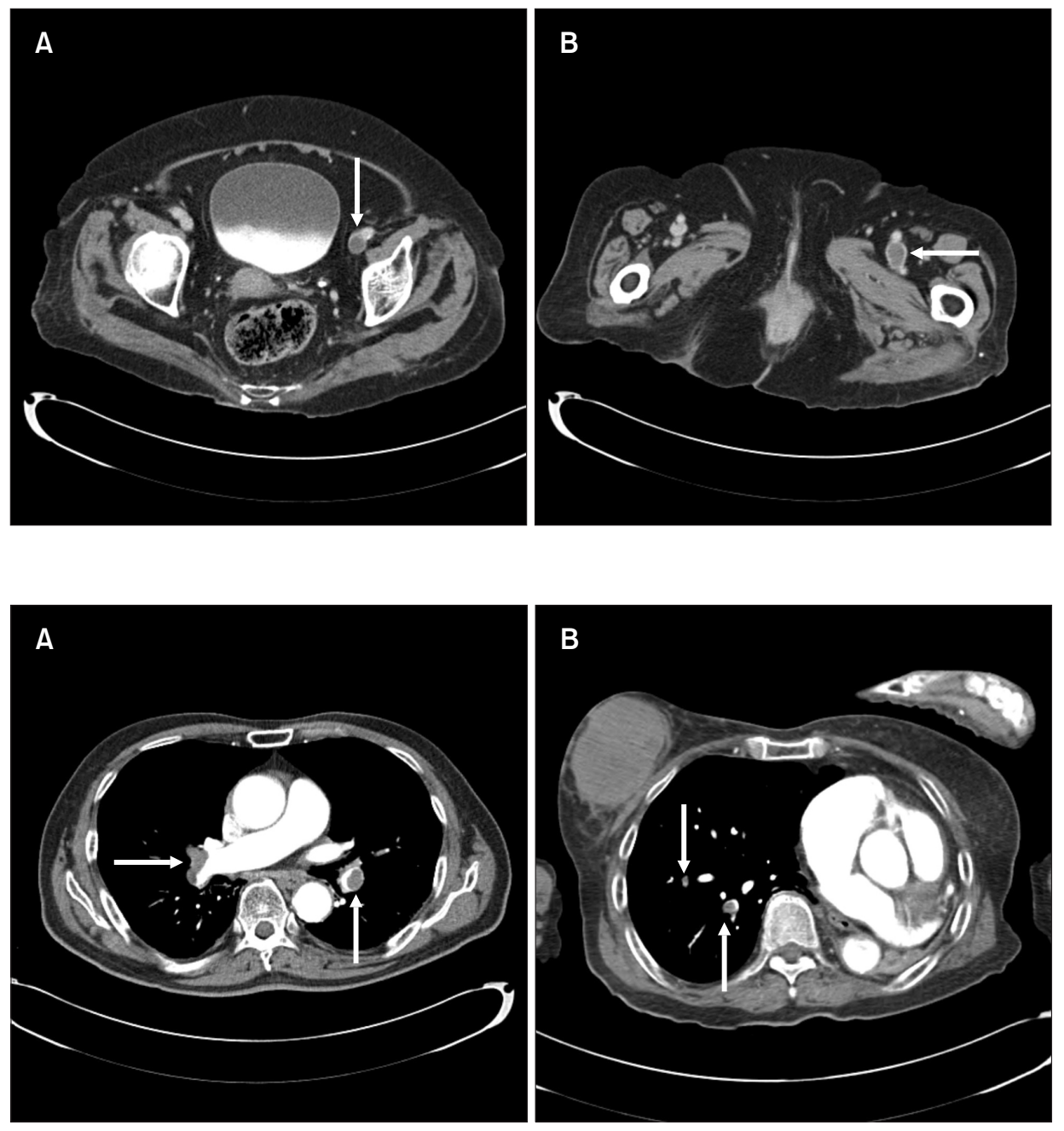

Fig. 1. Computed tomography angiograms of proximal deep vein thrombosis (DVT). (A) The arrow indicates DVT in the left external iliac vein. (B) The arrow indicates DVT in the left common femoral vein.

Fig. 2. Computed tomography angiograms of patients with pulmonary embolism (PE). (A) Bilateral PEs in the lobar arteries (arrows). (B) PE in the right segmental pulmonary arteries (arrows). 
brovascular accident, dementia, or COPD) for unfavorable outcomes and early all-cause mortality were evaluated.

A recent event was defined as any event that occurred within one month after a VTE diagnosis. VTE-related clinical outcomes were divided into unfavorable outcomes, major bleeding, and all-cause mortality. VTE-related unfavorable outcomes were defined when at least one of the following criteria was met: 1) hypotension (SBP <100 mmHg) or shock; 2) need for mechanical ventilation; 3) need for catecholamines to maintain organ perfusion; 4) need for cardiopulmonary resuscitation; and 5) all-cause death. Major bleeding was defined as life-threatening bleeding requiring transfusion of at least two units of packed red blood cells associated with a decrease in hemoglobin level $>2 \mathrm{~g} / \mathrm{dL}$ or the presence of retroperitoneal, intracranial, or intraocular bleeding. Massive PE was defined as PE associated with systemic hypotension (systolic blood pressure $<90 \mathrm{mmHg}$ ), PE requiring cardiopulmonary resuscitation, or the need for catecholamines.

Clinical outcomes such as unfavorable outcome, major bleeding, and 30-day all-cause mortality for patients with VTE were analyzed, and the clinical characteristics were compared between the DVT and PE groups using Fisher's exact test and the Chi-squared test. We also performed a univariate analysis of troponin 1 using enzyme immunoassay and d-dimer using enzyme-linked immunosorbent assay as risk factors for the development of unfavorable outcomes, major bleeding, and all-cause mortality.

Specified risk factors for unfavorable outcomes, major bleeding, and all-cause mortality within one month of diagnosis were analyzed using univariate and multiple logistic regression analyses. Candidate predictors $(\mathrm{P}<0.25$ after univariate analysis) and several variables possibly associated with VTE outcome were included in each multivariate regression analysis. All P-values were two-tailed. Statistical significance was considered at $\mathrm{P}<0.05$. All statistical analyses were performed using SPSS Statistics for Windows version 27 (IBM, Armonk, NY, USA).

Our study was approved by the Institutional Review Board of Seoul Medical Center (IRB no. 2021-05-001-002).

\section{RESULTS}

\section{1) Clinical characteristics and outcomes}

A total of 198 patients with VTE were enrolled, including 62 (31.3\%) patients with isolated DVT, 100 (50.5\%) with both DVT and PE, and 36 (18.2\%) with PE alone. In addition, 49 (24.7\%) had calf vein thrombosis and 113 (57.1\%) had proximal DVT. The mean age was $71.6 \pm 15.06$ years and

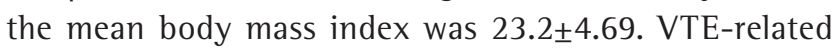
unfavorable outcomes occurred in 26 (13.1\%) patients, with a 30-day all-cause mortality of 17 (8.6\%) patients. Of 62 patients with isolated DVT, 7 (11.3\%) had unfavorable outcomes and 5 (8.1\%) had all-cause mortality. Among 100 patients with DVT and PE, 13 (13.0\%) had unfavorable outcomes and $8(8.0 \%)$ had all-cause mortality. Of 36 patients with PE alone, 6 (16.7\%) had unfavorable outcomes and 4

Table 1. Demographic features

\begin{tabular}{|c|c|c|c|c|}
\hline Demographic feature & All patient $(n=198)$ & Isolated DVT $(n=62,31.3 \%)$ & $P E(n=136,68.7 \%)$ & P-value ${ }^{a}$ \\
\hline Age (y) & $71.58 \pm 15.06$ & - & - & - \\
\hline$\leq 50$ & $19(9.6)$ & $6(9.7)$ & $13(9.6)$ & - \\
\hline $51-70$ & $55(27.8)$ & $20(32.3)$ & $35(25.7)$ & - \\
\hline$\geq 71$ & $124(62.6)$ & $36(58.1)$ & $88(64.7)$ & 0.370 \\
\hline Body mass index & $23.22 \pm 4.69$ & - & - & - \\
\hline$>25$ & $64(32.3)$ & $20(32.3)$ & $44(32.4)$ & 0.989 \\
\hline Sex, male & $78(39.4)$ & $25(40.3)$ & $53(39.0)$ & - \\
\hline \multicolumn{5}{|l|}{ Vital sign } \\
\hline Pulse rate $\geq 110 / \mathrm{min}$ & $27(13.6)$ & $3(4.8)$ & $24(17.6)$ & 0.014 \\
\hline Systolic blood pressure $<90 \mathrm{mmHg}$ & $20(10.1)$ & $6(9.7)$ & $14(10.3)$ & 0.894 \\
\hline Respiratory rate $\geq 30 / \mathrm{min}$ & $16(8.1)$ & $3(4.8)$ & $13(9.6)$ & 0.400 \\
\hline Body temperature $<36^{\circ} \mathrm{C}$ & $3(1.5)$ & $0(0.0)$ & $3(2.2)$ & 0.553 \\
\hline \multicolumn{5}{|l|}{ Risk factor for VTE } \\
\hline History of VTE & $16(8.1)$ & $5(8.1)$ & $11(8.1)$ & 0.995 \\
\hline Immobilization $\geq 3$ days & $106(53.5)$ & $40(64.5)$ & $66(48.5)$ & 0.036 \\
\hline Recent surgery $<4$ weeks & $53(26.8)$ & $25(40.3)$ & $28(20.6)$ & 0.004 \\
\hline Active malignancy and/or chemotherapy & $46(23.2)$ & $10(16.1)$ & $36(26.5)$ & 0.110 \\
\hline
\end{tabular}


Table 1. Continued

\begin{tabular}{|c|c|c|c|c|}
\hline Demographic feature & All patient $(n=198)$ & Isolated DVT $(n=62,31.3 \%)$ & $\operatorname{PE}(n=136,68.7 \%)$ & P-value ${ }^{a}$ \\
\hline \multicolumn{5}{|l|}{ Comorbidities } \\
\hline Hypertension & $114(57.6)$ & $36(58.1)$ & $78(57.4)$ & 0.925 \\
\hline Diabetes mellitus & $57(28.8)$ & $17(27.4)$ & $40(29.4)$ & 0.774 \\
\hline Coronary artery disease & $16(8.1)$ & $3(4.8)$ & $13(9.6)$ & 0.400 \\
\hline Chronic kidney disease & $9(4.5)$ & $3(4.8)$ & $6(4.4)$ & $>0.999$ \\
\hline Chronic heart failure & $11(5.6)$ & $1(1.6)$ & $10(7.4)$ & 0.178 \\
\hline Smoking & $29(14.6)$ & $13(21.0)$ & $16(11.8)$ & 0.089 \\
\hline Pneumonia & $38(19.2)$ & $6(9.7)$ & $32(23.5)$ & - \\
\hline Chronic obstructive pulmonary disease & $18(9.1)$ & $1(1.6)$ & $17(12.5)$ & 0.014 \\
\hline All pulmonary disease & $56(28.3)$ & $8(12.9)$ & $48(35.3)$ & 0.001 \\
\hline Cerebrovascular accident & $41(20.7)$ & $12(19.4)$ & $29(21.3)$ & - \\
\hline Dementia & $29(14.6)$ & $9(14.5)$ & $20(14.7)$ & - \\
\hline \multicolumn{5}{|l|}{ Location of PE } \\
\hline Main \& lobar arteries & $2(1.0)$ & $0(0.0)$ & $2(1.5)$ & - \\
\hline Segmental \& subsegmental arteries & $45(22.7)$ & $0(0.0)$ & $45(33.1)$ & - \\
\hline Massive PE & $24(12.1)$ & $7(11.3)$ & $17(12.5)$ & 0.809 \\
\hline \multicolumn{5}{|l|}{ Location of DVT } \\
\hline Distal & $49(24.7)$ & $16(25.8)$ & $33(24.3)$ & - \\
\hline Proximal & $113(57.1)$ & $46(74.2)$ & $67(49.3)$ & - \\
\hline High sPESI & $124(62.6)$ & $29(46.8)$ & $95(69.9)$ & 0.002 \\
\hline Inferior vena cava filter insertion & $40(20.2)$ & $15(24.2)$ & $25(18.4)$ & 0.345 \\
\hline Anticoagulation treatment & $185(93.4)$ & $55(88.7)$ & $130(95.6)$ & 0.070 \\
\hline Novel oral anticoagulants & $122(61.6)$ & $32(51.6)$ & $90(66.2)$ & 0.051 \\
\hline Need for mechanical ventilation & $8(4.0)$ & $2(3.2)$ & $6(4.4)$ & $>0.999$ \\
\hline Need for inotropics & $14(7.1)$ & $4(6.5)$ & $10(7.4)$ & $>0.999$ \\
\hline Need for thrombolysis or thrombectomy & $1(0.5)$ & $0(0.0)$ & $1(0.7)$ & - \\
\hline Cardiopulmonary resuscitation & $3(1.5)$ & $0(0.0)$ & $3(2.2)$ & 0.553 \\
\hline Unfavorable outcome & $26(13.1)$ & $7(11.3)$ & $19(14.0)$ & 0.605 \\
\hline Major bleeding & $6(3.0)$ & $2(3.2)$ & $4(2.9)$ & $>0.999$ \\
\hline PE-related death & $3(1.5)$ & $0(0.0)$ & $3(2.2)$ & 0.553 \\
\hline \multirow[t]{2}{*}{ All-cause mortality } & $17(8.6)$ & $5(8.1)$ & $12(8.8)$ & 0.860 \\
\hline & Total $(n=133)$ & DVT only $(n=31,23.3 \%)$ & $P E_{ \pm} D V T(n=102,76.7 \%)$ & \\
\hline \multirow[t]{2}{*}{ Arterial saturation $<90 \%$} & $30(22.6)$ & $7(22.6)$ & $23(22.5)$ & - \\
\hline & Total $(n=158)$ & DVT only $(n=41,25.9 \%)$ & $P E \pm D V T(n=117,74.1 \%)$ & \\
\hline \multirow[t]{2}{*}{ Elevated D-dimer } & $151(95.6)$ & $38(92.7)$ & $113(96.6)$ & - \\
\hline & Total $(n=129)$ & DVT only $(n=31,24.0 \%)$ & $P E \pm D V T(n=98,76.0 \%)$ & \\
\hline Elevated troponin I & 36 (27.9) & $7(22.6)$ & $29(29.6)$ & - \\
\hline
\end{tabular}

Values are presented as mean \pm standard deviation or number (\%).

DVT, deep venous thrombosis; PE, pulmonary embolism; VTE, venous thromboembolism; sPESI, simplified pulmonary embolism severity score; -, not available.

${ }^{a}$ Chi-squared test or Fisher exact test, logistic regression model.

(11.1\%) had all-cause mortality.

Several clinical characteristics showed statistically significant differences between the DVT and PE groups (Table 1). The prevalence of those with immobilization $\geq 3$ days, recent surgery $\leq 4$ weeks, the presence of COPD, pulse $\geq 110$ / min, and a high sPESI was significantly higher in the PE group. Unfavorable outcomes (11.3\% in the DVT group vs. $14.0 \%$ in the PE group, $\mathrm{P}=0.605$ ) and all-cause mortality (8.1\% vs. $8.8 \%, P=0.860$ ) were lower in the DVT group than in the PE group, but the difference was not significant (Table 
Table 2. Characteristics of patients with unfavorable outcomes $(n=26)$

\begin{tabular}{|c|c|c|c|c|}
\hline \multirow{2}{*}{ Risk factor } & \multirow{2}{*}{$\begin{array}{c}\text { Unfavorable outcome } \\
(n=26,13.1 \%)\end{array}$} & \multirow{2}{*}{$\begin{array}{l}\text { Univariate } \\
\text { P-value }^{\mathrm{a}}\end{array}$} & \multicolumn{2}{|c|}{ Multivariate } \\
\hline & & & P-value ${ }^{a}$ & $95 \% \mathrm{Cl}$ \\
\hline Age (y) & $74.5 \pm 13.2$ & 0.449 & - & - \\
\hline$\geq 71$ & $19(73.1)$ & - & 0.305 & $0.566-6.169$ \\
\hline Body mass index & $22.55 \pm 7.94$ & - & - & - \\
\hline$>25$ & $5(19.2)$ & 0.126 & 0.890 & $0.255-3.277$ \\
\hline Symptom of DVT and PE & $20(76.9)$ & 0.227 & 0.415 & $0.501-5.343$ \\
\hline Subjective leg symptom (edema) & $10(38.5)$ & 0.650 & - & - \\
\hline Subjective chest symptom & $11(42.3)$ & 0.338 & - & - \\
\hline \multicolumn{5}{|l|}{ Risk factor for VTE } \\
\hline History of VTE & $2(7.7)$ & $>0.999$ & - & - \\
\hline Immobilization $\geq 3 \mathrm{~d}$ & $19(73.1)$ & 0.032 & 0.092 & $0.849-8.644$ \\
\hline Recent surgery $<4$ wk & $7(26.9)$ & 0.985 & - & - \\
\hline Active malignancy and/or chemotherapy & $8(30.8)$ & 0.329 & 0.457 & $0.464-5.518$ \\
\hline \multicolumn{5}{|l|}{ Comorbidities } \\
\hline Hypertension & $15(57.7)$ & 0.990 & - & - \\
\hline Diabetes mellitus & $11(42.3)$ & 0.102 & 0.637 & $0.443-3.777$ \\
\hline Coronary artery disease & $3(11.5)$ & 0.447 & 0.228 & $0.544-12.774$ \\
\hline Chronic kidney disease & $2(7.7)$ & 0.336 & - & - \\
\hline Chronic heart failure & $2(7.7)$ & 0.641 & - & - \\
\hline Smoking & $5(19.2)$ & 0.478 & - & - \\
\hline Pneumonia & $6(23.1)$ & 0.589 & - & - \\
\hline Chronic obstructive pulmonary disease & $4(15.4)$ & 0.266 & 0.456 & $0.082-3.072$ \\
\hline All pulmonary disease & $11(42.3)$ & 0.102 & - & - \\
\hline Cerebrovascular accident & $4(15.4)$ & 0.608 & - & - \\
\hline Dementia & $6(23.1)$ & 0.192 & 0.385 & $0.118-2.285$ \\
\hline \multicolumn{5}{|l|}{ Vital sign } \\
\hline Pulse rate $\geq 110 / \mathrm{min}$ & $13(50.0)$ & 0.001 & $<0.001$ & $3.418-44.744$ \\
\hline Systolic blood pressure $<90 \mathrm{mmHg}$ & $20(76.9)$ & 0.001 & - & - \\
\hline Respiratory rate $\geq 30 / \mathrm{min}$ & $8(30.8)$ & 0.001 & 0.013 & $1.429-21.392$ \\
\hline Body temperature $<36^{\circ} \mathrm{C}$ & $2(7.7)$ & 0.046 & 0.512 & $0.108-86.780$ \\
\hline \multicolumn{5}{|l|}{ Types of VTE } \\
\hline Isolated DVT & $7(26.9)$ & 0.605 & - & - \\
\hline PE & 19 (73.1) & 0.605 & - & - \\
\hline Inferior vena cava filter insertion & $6(23.1)$ & 0.695 & - & - \\
\hline Anticoagulation treatment & $23(88.8)$ & 0.385 & 0.075 & $0.050-1.155$ \\
\hline \multicolumn{5}{|c|}{$(n=23,12.4 \%)$} \\
\hline Novel oral anticoagulants (total $n=185$ ) & $14(60.9)$ & 0.583 & - & - \\
\hline \multicolumn{5}{|c|}{$(n=24,18.0 \%)$} \\
\hline Arterial saturation $<90 \%$ (total $n=133$ ) & $12(50.0)$ & 0.001 & - & - \\
\hline \multicolumn{5}{|c|}{$(n=22,13.9 \%)$} \\
\hline Elevated D-dimer (total $n=158$ ) & $22(100.0)$ & 0.594 & - & - \\
\hline \multicolumn{5}{|c|}{$(n=24,18.6 \%)$} \\
\hline Elevated troponin I (total $n=129$ ) & $11(45.8)$ & 0.030 & - & - \\
\hline
\end{tabular}

Values are presented as mean \pm standard deviation or number (\%).

$\mathrm{Cl}$, confidence interval; DVT, deep venous thrombosis; PE, pulmonary embolism; VTE, venous thromboembolism; -, not available.

${ }^{a}$ Chi-squared test or Fisher exact test, logistic regression model. 
1). Among 46 patients with malignancy \pm chemotherapy, lung cancer was the most common malignancy $(n=11$ [23.9\%]), and the prevalence of PE was higher than that of DVT (78.3\% vs. $21.7 \%)$. However, the difference between the two groups was not statistically significant $(\mathrm{P}=0.110)$.

\section{2) Predictors for unfavorable outcome}

VTE-related unfavorable outcomes were observed in 26 $(13.1 \%)$ patients. Among the 16 patients with a respiratory rate $\geq 30 / \mathrm{min}, 8$ (50.0\%) showed an unfavorable outcome (Table 2). Univariate analysis of risk factors revealed that immobilization $\geq 3$ days $(\mathrm{P}=0.032)$, pulse $\geq 110 / \mathrm{min}(\mathrm{P}=0.001)$, respiratory rate $\geq 30 / \mathrm{min}(\mathrm{P}=0.001)$, and temperature $<36^{\circ} \mathrm{C}$ $(\mathrm{P}=0.046)$ were statistically significant risk factors (Table 2$)$. In addition, the troponin 1 test was performed in 129 patients, and an elevated level was identified as a statistically significant factor for VTE-related unfavorable outcomes in the univariate analysis $(\mathrm{P}=0.030)$. We obtained arterial blood gas analysis data for 133 of 198 patients with VTE. An arterial saturation $<90 \%$ was statistically significant in the univariate analysis $(\mathrm{P}=0.001)$. However, the presence of coronary artery disease and congestive heart failure, VTE type, VTE location, anticoagulation treatment, and elevated d-dimer levels were not significantly associated. The multivariate analysis revealed that pulse $\geq 110 /$ min (odds ratio [OR], 12.4; 95\% confidence interval [Cl] [6], 3.4-44.7; $\mathrm{P}=0.001)$ and respiratory rate $\geq 30 / \mathrm{min}(\mathrm{OR}, 5.5 ; 95 \% \mathrm{Cl}$, 1.4-21.4; $\mathrm{P}=0.013$ ) were statistically significant predictors of VTE-related unfavorable outcomes (Table 2).

\section{3) Risk factors for major bleeding}

Major bleeding occurred in $6(3.0 \%)$ patients (Table 3). The major bleeding rate in patients with a history of recent surgery $\leq 4$ weeks was higher than that in patients without a history $(4 / 53[7.5 \%]$ vs. $2 / 145[1.4 \%])$. With regard to recent surgery, hip surgery was the most common ( $n=14$ [26.4\%]), followed by spine surgery ( $\mathrm{n}=9$ [17.0\%]). Major bleeding occurred in the brain, hip joint, and stomach in each of those two cases. Risk factors for major bleeding were subjected to univariate analysis, and a history of recent surgery was statistically significant $(\mathrm{P}=0.045)$. However, in the multivariate analysis, diabetes was statistically significant $(\mathrm{P}=0.043)$.

\section{4) Predictors for all-cause mortality}

All-cause mortality was observed in 17 patients (8.6\%). A history of malignancy \pm chemotherapy was present in 46 of 198 patients. Lung cancer was the most common $(n=11$ [23.9\%]), followed by colon cancer $(n=8[17.4 \%])$. Stage IV cancer was the most common $(n=14$ [30.4\%]). Among the 24 patients with massive PE, all-cause mortality occurred in $10(41.7 \%)$. Among the 124 patients with high sPESI, all-cause mortality occurred in 15 (12.1\%). Anticoagulation treatment was administered to 185 (93.4\%). All-cause mortality occurred in $4(30.8 \%)$ patients not treated with anticoagulants and in $13(7.0 \%)$ patients treated with anticoagulants. In addition, 6 of 8 (75.0\%) patients who needed mechanical ventilation died within 30 days of their hospital stay. The univariate analysis revealed no NOACs, arterial

Table 3. Characteristics of patients with major bleeding $(n=6)$

\begin{tabular}{|c|c|c|c|c|}
\hline Risk factor & $\begin{array}{l}\text { Major bleeding } \\
(\mathrm{n}=6,3.0 \%)\end{array}$ & $\begin{array}{c}\text { Univariate } \\
\text { P-value }^{\mathrm{a}}\end{array}$ & $\begin{array}{c}\text { Multivariate } \\
\text { P-value }^{\mathrm{a}}\end{array}$ & $95 \% \mathrm{Cl}$ \\
\hline Age (y) & $71.83 \pm 16.52$ & 0.364 & - & - \\
\hline$\leq 50$ & $0(0.0)$ & - & - & - \\
\hline $51-70$ & $3(50.0)$ & - & - & - \\
\hline$\geq 71$ & $3(50.0)$ & - & - & - \\
\hline Body mass index & $21.87 \pm 1.93$ & - & - & - \\
\hline \multirow[t]{2}{*}{$>25$} & $0(0.0)$ & 0.180 & 0.996 & - \\
\hline & $(n=5,2.5 \%)$ & & & \\
\hline Symptom of DVT and PE (total $n=197$ ) & $3(60.0)$ & $>0.999$ & - & - \\
\hline Subjective leg symptom (edema) & $1(20.0)$ & 0.661 & - & - \\
\hline Subjective chest symptom & $2(40.0)$ & $>0.999$ & - & - \\
\hline \multicolumn{5}{|l|}{ Risk factor for VTE } \\
\hline History of VTE & $0(0.0)$ & $>0.999$ & - & - \\
\hline Immobilization $\geq 3 \mathrm{~d}$ & $5(83.3)$ & 0.219 & 0.642 & $0.132-26.737$ \\
\hline Recent surgery $<4$ wk & $4(66.7)$ & 0.045 & 0.254 & $0.407-29.980$ \\
\hline Active malignancy and/or chemotherapy & $1(16.7)$ & $>0.999$ & - & - \\
\hline
\end{tabular}


Table 3. Continued

\begin{tabular}{|c|c|c|c|c|}
\hline Risk factor & $\begin{array}{l}\text { Major bleeding } \\
(n=6,3.0 \%)\end{array}$ & $\begin{array}{c}\text { Univariate } \\
\text { P-value }^{\mathrm{a}}\end{array}$ & $\begin{array}{c}\text { Multivariate } \\
\text { P-value }^{\mathrm{a}}\end{array}$ & $95 \% \mathrm{Cl}$ \\
\hline \multicolumn{5}{|l|}{ Comorbidities } \\
\hline Hypertension & $2(33.3)$ & 0.404 & - & - \\
\hline Diabetes mellitus & $4(66.7)$ & 0.058 & 0.043 & $1.074-68.319$ \\
\hline Coronary artery disease & $1(16.7)$ & 0.401 & - & - \\
\hline Chronic kidney disease & $0(0.0)$ & $>0.999$ & - & - \\
\hline Chronic heart failure & $0(00)$ & $>0.999$ & - & - \\
\hline Smoking & $1(16.7)$ & $>0.999$ & - & - \\
\hline Pneumonia & $2(33.3)$ & 0.325 & - & - \\
\hline Chronic obstructive pulmonary disease & $0(0.0)$ & $>0.999$ & - & - \\
\hline All pulmonary disease & $2(33.3)$ & $>0.999$ & - & - \\
\hline Cerebrovascular accident & $2(33.3)$ & 0.606 & - & - \\
\hline Dementia & $1(16.7)$ & $>0.999$ & - & - \\
\hline \multicolumn{5}{|l|}{ Vital sign } \\
\hline Pulse rate $\geq 110 / \mathrm{min}$ & $1(16.7)$ & 0.590 & - & - \\
\hline Systolic blood pressure $<90 \mathrm{mmHg}$ & $1(16.7)$ & 0.477 & - & - \\
\hline Respiratory rate $\geq 30 / \mathrm{min}$ & $2(33.3)$ & 0.076 & 0.113 & $0.589-147.536$ \\
\hline Body temperature $<36^{\circ} \mathrm{C}$ & $0(0.0)$ & $>0.999$ & - & - \\
\hline \multicolumn{5}{|l|}{ Types of VTE } \\
\hline Isolated DVT & $2(33.3)$ & $>0.999$ & - & - \\
\hline $\mathrm{PE}$ & $4(66.7)$ & $>0.999$ & - & - \\
\hline Inferior vena cava filter insertion & $3(50.0)$ & 0.098 & 0.089 & $0.702-140.721$ \\
\hline Anticoagulation treatment & $5(83.3)$ & 0.338 & 0.379 & $0.017-4.677$ \\
\hline \multicolumn{5}{|c|}{$(n=5,2.7 \%)$} \\
\hline Novel oral anticoagulants (total $n=185$ ) & $3(60.0)$ & $>0.999$ & - & - \\
\hline \multicolumn{5}{|c|}{$(n=6,4.5 \%)$} \\
\hline Arterial saturation $<90 \%$ (total $n=133$ ) & $2(33.3)$ & 0.617 & - & - \\
\hline \multicolumn{5}{|c|}{$(n=4,2.5 \%)$} \\
\hline Elevated D-dimer (total $n=158$ ) & $4(100.0)$ & $>0.999$ & - & - \\
\hline \multicolumn{5}{|c|}{$(n=4,3.1 \%)$} \\
\hline Elevated troponin I (total $n=129$ ) & $0(0.0)$ & 0.576 & - & - \\
\hline
\end{tabular}

Values are presented as mean \pm standard deviation or number (\%).

$\mathrm{Cl}$, confidence interval; DVT, deep venous thrombosis; PE, pulmonary embolism; VTE, venous thromboembolism; -, not available.

${ }^{a}$ Chi-squared test or Fisher exact test, logistic regression model.

saturation $<90 \%$, history of malignancy \pm chemotherapy, pulse $\geq 110 / \mathrm{min}$, systolic blood pressure $<90 \mathrm{mmHg}$, respiratory rate $\geq 30 / \mathrm{min}$, massive PE, high $\mathrm{SPESl}$, anticoagulation treatment, need for mechanical ventilation, need for inotropics, and cardiopulmonary resuscitation were risk factors for all-cause mortality (Table 4). Multivariate regression analysis showed that a history of malignancy \pm chemotherapy (OR, 7.38; 95\% Cl, 1.219-44.681; $\mathrm{P}=0.030$ ), anticoagulation treatment (OR, 0.061; 95\% Cl, 0.006-0.590; $\mathrm{P}=0.016)$, and need for mechanical ventilation (OR, 235.220; 95\% $\mathrm{Cl}, 4.954-11168.024 ; \mathrm{P}=0.006)$ were statistically significant predictors of all-cause mortality (Table 4).

\section{DISCUSSION}

VTE, including DVT and PE, is common in hospitalized patients. DVT and PE have the same disease processes but different clinical manifestations. However, few studies have reported the overall clinical outcomes of VTE. Despite recent advances in medicine, the 30-day all-cause mortality of VTE remains high around $8 \%$ to $11 \%$ [9,11]. Our study findings also indicated that the 30-day all-cause mortality rate was relatively high (8.6\%). Tagalakis et al. [11] reported that the 30-day mortality rate after VTE was $10.6 \%$. The all-cause mortality rates of DVT and PE were similar (8.1\% 


\section{vs. $8.8 \%)$.}

Our multivariate analysis showed that a high pulse and respiratory rate were statistically significant predictors of unfavorable outcomes. As expected, tachycardia and tachypnea were early signs of shock and cardiopulmonary resuscitation. This should be interpreted as physicians employing aggressive intervention for tachycardia and tachypnea in VTE patients to prevent unfavorable outcomes.

In several randomized controlled trials, the incidence of major bleeding at 3 to 6 months is as high as 4\% [12,13]. In the present study, 30-day major bleeding occurred in 6 of 198 (3.0\%) of the enrolled patients, a rate slightly lower than that reported in previous studies. Of the 185 patients who underwent anticoagulation treatment, $5(2.7 \%)$ had major bleeding. Our multivariate analysis showed that diabetes mellitus was the only predictor of VTE-related major bleeding. The present study found that major bleeding was not associated with all-cause mortality.

Several studies have reported that increased age is associated with mortality [14,15]. In our study, 14 of 124 (11.3\%) patients older than 70 years and 3 of $74(4.1 \%)$ patients younger than 70 years died; the difference was not statistically significant. Those with a systolic blood pressure $<90$ $\mathrm{mmHg}$ at the initial event showed a higher all-cause mortality rate (30.0\% vs. $6.2 \%)$. In addition, massive PE (41.7\%), high SPESI (12.1\%), and a respiratory rate $>30 / \mathrm{min}(30.0 \%)$

Table 4. Demographics of all-cause mortality $(n=17)$

\begin{tabular}{|c|c|c|c|c|}
\hline Risk factor & $\begin{array}{l}\text { All-cause mortality } \\
\qquad(n=17,8.6 \%)\end{array}$ & $\begin{array}{l}\text { Univariate } \\
\text { P-value }^{a}\end{array}$ & $\begin{array}{c}\text { Multivariate } \\
\text { P-value }^{a}\end{array}$ & $95 \% \mathrm{Cl}$ \\
\hline Age (y) & $76.24 \pm 14.83$ & 0.24 & - & - \\
\hline$\leq 50$ & $1(5.9)$ & - & - & - \\
\hline $51-70$ & $2(11.8)$ & - & - & - \\
\hline$\geq 71$ & $14(82.4)$ & - & 0.242 & $0.376-48.124$ \\
\hline Body mass index & $22.36 \pm 5.19$ & - & - & - \\
\hline$>25$ & $3(17.6)$ & 0.277 & - & - \\
\hline \multicolumn{5}{|c|}{$(n=16,8.1 \%)$} \\
\hline Symptom of DVT and PE & 11 (68.8) & 0.842 & - & - \\
\hline Subjective leg symptom (edema) & 7 (43.8) & 0.418 & - & - \\
\hline Subjective chest symptom & $5(31.3)$ & 0.808 & - & - \\
\hline \multicolumn{5}{|l|}{ Risk factor for VTE } \\
\hline History of VTE & $2(11.8)$ & 0.633 & - & - \\
\hline Immobilization $\geq 3 \mathrm{~d}$ & $10(58.8)$ & 0.648 & - & - \\
\hline Recent surgery $<4$ wk & $3(17.6)$ & 0.568 & - & - \\
\hline Active malignancy and/or chemotherapy & $8(47.1)$ & 0.015 & 0.030 & $1.219-44.681$ \\
\hline \multicolumn{5}{|l|}{ Comorbidities } \\
\hline Hypertension & $12(70.6)$ & 0.256 & - & - \\
\hline Diabetes mellitus & 5 (29.4) & 0.953 & - & - \\
\hline Coronary artery disease & $3(17.6)$ & 0.146 & 0.070 & $0.847-48.882$ \\
\hline Chronic kidney disease & $1(5.9)$ & 0.562 & - & - \\
\hline Chronic heart failure & $3(17.6)$ & 0.057 & 0.299 & $0.321-40.396$ \\
\hline Smoking & $4(23.5)$ & 0.284 & - & - \\
\hline Pneumonia & $4(23.5)$ & 0.747 & - & - \\
\hline Chronic obstructive pulmonary disease & $3(17.6)$ & 0.190 & $>0.999$ & $0.084-11.891$ \\
\hline All pulmonary disease & $6(35.3)$ & 0.535 & - & - \\
\hline Cerebrovascular accident & $1(5.9)$ & 0.206 & 0.071 & $0.003-1.272$ \\
\hline Dementia & $4(23.5)$ & 0.284 & - & - \\
\hline \multicolumn{5}{|l|}{ Vital sign } \\
\hline Pulse rate $\geq 110 / \mathrm{min}$ & $7(41.2)$ & 0.001 & 0.484 & $0.180-37.384$ \\
\hline Systolic blood pressure $<90 \mathrm{mmHg}$ & $6(35.3)$ & 0.001 & - & - \\
\hline Respiratory rate $\geq 30 / \mathrm{min}$ & $5(29.4)$ & 0.001 & 0.213 & $0.466-30.763$ \\
\hline Body temperature $<36^{\circ} \mathrm{C}$ & $1(5.9)$ & 0.237 & 0.783 & $0.000-8562.725$ \\
\hline
\end{tabular}


Table 4. Continued

\begin{tabular}{|c|c|c|c|c|}
\hline Risk factor & $\begin{array}{c}\text { All-cause mortality } \\
\quad(n=17,8.6 \%)\end{array}$ & $\begin{array}{c}\text { Univariate } \\
\text { P-value }^{\mathrm{a}}\end{array}$ & $\frac{\text { Multivariate }}{\text { P-value }^{\mathrm{a}}}$ & $95 \% \mathrm{Cl}$ \\
\hline \multicolumn{5}{|l|}{ Types of VTE } \\
\hline Isolated DVT & $5(29.4)$ & 0.860 & - & - \\
\hline PE & $12(70.6)$ & 0.860 & - & - \\
\hline Massive PE & $10(58.8)$ & 0.001 & 0.795 & $0.047-54.305$ \\
\hline High sPESI & $15(88.2)$ & 0.033 & - & - \\
\hline Inferior vena cava filter insertion & $3(17.6)$ & $>0.999$ & - & - \\
\hline Anticoagulation treatment & $13(76.5)$ & 0.017 & 0.016 & $0.006-0.590$ \\
\hline Novel oral anticoagulants & $4(23.5)$ & 0.583 & - & - \\
\hline Need for mechanical ventilation & $6(35.3)$ & 0.001 & 0.006 & 4.954-11168.024 \\
\hline Need for inotropics & $8(47.1)$ & 0.001 & 0.754 & $0.061-47.367$ \\
\hline Need for thrombolysis or thrombectomy & $0(0.0)$ & $>0.999$ & - & - \\
\hline Cardiopulmonary resuscitation & $2(11.8)$ & 0.020 & 0.291 & $0.098-2310.987$ \\
\hline Major bleeding & $1(5.9)$ & 0.421 & - & - \\
\hline Arterial saturation $<90 \%$ & $9(60.0)$ & 0.001 & - & - \\
\hline Elevated D-dimer & $15(100.0)$ & $>0.999$ & - & - \\
\hline Elevated troponin I & $7(50.0)$ & 0.051 & - & - \\
\hline
\end{tabular}

Values are presented as mean \pm standard deviation or number (\%).

$\mathrm{Cl}$, confidence interval; DVT, deep venous thrombosis; PE, pulmonary embolism; VTE, venous thromboembolism; sPESI, simplified pulmonary embolism severity score; -, not available.

${ }^{\mathrm{a}}$ Chi-squared test or Fisher exact test, logistic regression model.

had higher all-cause mortality rates. Regarding the anticoagulant treatment strategies, the NOAC group showed a lower mortality rate than the other anticoagulant groups (3.3\% vs. $14.3 \%)$. The better results in the NOAC versus vitamin $\mathrm{K}$ antagonist group are thought to be attributed to the convenience of use, minor drug and food interactions, consistent pharmacokinetics and pharmacodynamics, and good compliance [16]. The multivariate regression analysis showed that a history of malignancy \pm chemotherapy, anticoagulation treatment, and need for mechanical ventilation were statistically significant predictors of all-cause mortality. This group of patients should be monitored closely, and aggressive interventions are needed to prevent mortality.

\section{CONCLUSION}

VTE-related mortality and morbidity rates remained high (8.1\%-8.8\%). In cases of tachycardia and tachypnea, early aggressive treatment is needed to prevent unfavorable outcomes. Patients with a history of malignancy, no anticoagulation use, and need for mechanical ventilation should be monitored closely to prevent mortality.

\section{FUNDING}

None.

\section{CONFLICTS OF INTEREST}

The authors have nothing to disclose.

\section{ORCID}

Han Young Lee

https://orcid.org/0000-0002-6509-4154

Tae Hoon Yeo

https://orcid.org/0000-0002-6705-3540

Tae Kyung Heo

https://orcid.org/0000-0001-8275-2377

Young Gyu Cho

https://orcid.org/0000-0001-9665-0945

Dong Hui Cho

https://orcid.org/0000-0003-4515-521X

Kyung Bok Lee

https://orcid.org/0000-0003-1111-118X

\section{AUTHOR CONTRIBUTIONS}

Conception and design: HYL, KBL. Analysis and interpretation: HYL, KBL. Data Collection: THY, TKH. Writing the article: HYL, KBL. Critical revision of the article: YGC, DHC. Final approval of the article: all authors. Statistical analysis: KBL. Obtained funding: None. Overall responsibility: KBL 
1) Schulman S, Ageno W, Konstantinides SV. Venous thromboembolism: past, present and future. Thromb Haemost 2017;117:1219-1229.

2) Tritschler T, Kraaijpoel N, Le Gal G, Wells PS. Venous thromboembolism: advances in diagnosis and treatment. JAMA 2018;320:1583-1594.

3) Arcelus Jl, Caprini JA, Monreal M, Suárez C, González-Fajardo J. The management and outcome of acute venous thromboembolism: a prospective registry including 4011 patients. J Vasc Surg 2003;38:916-922.

4) Anderson FA Jr, Spencer FA. Risk factors for venous thromboembolism. Circulation 2003;107(23 Suppl 1):19116.

5) Nakamura M, Fujioka H, Yamada N, Sakuma M, Okada O, Nakanishi N, et al. Clinical characteristics of acute pulmonary thromboembolism in Japan: results of a multicenter registry in the Japanese Society of Pulmonary Embolism Research. Clin Cardiol 2001;24:132-138.

6) Bhatt M, Braun C, Patel P, Patel P, Begum $\mathrm{H}$, Wiercioch $\mathrm{W}$, et al. Diagnosis of deep vein thrombosis of the lower extremity: a systematic review and meta-analysis of test accuracy. Blood Adv 2020;4:1250-1264.
7) Hyers TM, Agnelli G, Hull RD, Morris TA, Samama M, Tapson V, et al. Antithrombotic therapy for venous thromboembolic disease. Chest 2001;119(1 Suppl):176S-193S.

8) Kearon C, Akl EA, Ornelas J, Blaivas A, Jimenez D, Bounameaux $\mathrm{H}$, et al. Antithrombotic therapy for VTE disease: CHEST guideline and expert panel report. Chest 2016;149:315-352.

9) Nakamura M, Miyata T, Ozeki Y, Takayama M, Komori K, Yamada N, et al. Current venous thromboembolism management and outcomes in Japan. Circ J 2014;78:708-717.

10) Spirk D, Husmann M, Hayoz D, Baldi T, Frauchiger B, Engelberger R, et al. Predictors of in-hospital mortality in elderly patients with acute venous thrombo-embolism: the SWlss Venous ThromboEmbolism Registry (SWIVTER). Eur Heart J 2012;33:921-926.

11) Tagalakis V, Patenaude V, Kahn SR, Suissa $\mathrm{S}$. Incidence of and mortality from venous thromboembolism in a realworld population: the Q-VTE Study Cohort. Am J Med 2013;126:832.e13832.e21.

12) Fiessinger JN, Huisman MV, Davidson BL, Bounameaux H, Francis CW, Eriksson $\mathrm{H}$, et al. Ximelagatran vs lowmolecular-weight heparin and war- farin for the treatment of deep vein thrombosis: a randomized trial. JAMA 2005;293:681-689.

13) Büller HR, Davidson BL, Decousus H, Gallus A, Gent M, Piovella F, et al. Fondaparinux or enoxaparin for the initial treatment of symptomatic deep venous thrombosis: a randomized trial. Ann lntern Med 2004;140:867873.

14) Heit JA, Silverstein MD, Mohr DN, Petterson TM, O'Fallon WM, Melton LJ 3rd. Predictors of survival after deep vein thrombosis and pulmonary embolism: a population-based, cohort study. Arch Intern Med 1999;159:445453.

15) Andresen MS, Sandven I, Brunborg C, Njaastad AM, Strekerud F, Abdelnoor $\mathrm{M}$, et al. Mortality and recurrence after treatment of VTE: long term follow-up of patients with good life-expectancy. Thromb Res 2011;127:540546.

16) Mekaj YH, Mekaj AY, Duci SB, Miftari El. New oral anticoagulants: their advantages and disadvantages compared with vitamin $\mathrm{K}$ antagonists in the prevention and treatment of patients with thromboembolic events. Ther Clin Risk Manag 2015;11:967-977. 